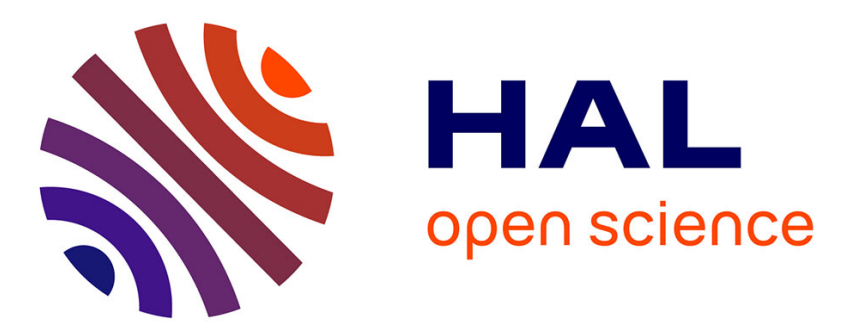

\title{
Model-Based Generation of Synthetic 3D Time-Lapse Sequences of Motile Cells with Growing Filopodia
}

Dmitry V Sorokin, Igor Peterlik, Vladimír V Ulman, David Svoboda, Martin Maška

\section{- To cite this version:}

Dmitry V Sorokin, Igor Peterlik, Vladimír V Ulman, David Svoboda, Martin Maška. Model-Based Generation of Synthetic 3D Time-Lapse Sequences of Motile Cells with Growing Filopodia. International Symposium on Biomedical Imaging, Apr 2017, Melbourne, Australia. hal-01445488

\section{HAL Id: hal-01445488 \\ https://inria.hal.science/hal-01445488}

Submitted on 24 Jan 2017

HAL is a multi-disciplinary open access archive for the deposit and dissemination of scientific research documents, whether they are published or not. The documents may come from teaching and research institutions in France or abroad, or from public or private research centers.
L'archive ouverte pluridisciplinaire HAL, est destinée au dépôt et à la diffusion de documents scientifiques de niveau recherche, publiés ou non, émanant des établissements d'enseignement et de recherche français ou étrangers, des laboratoires publics ou privés. 


\title{
MODEL-BASED GENERATION OF SYNTHETIC 3D TIME-LAPSE SEQUENCES OF MOTILE CELLS WITH GROWING FILOPODIA
}

\author{
Dmitry V. Sorokin ${ }^{\star \dagger} \quad$ Igor Peterlík $k^{\ddagger *} \quad$ Vladimír Ulman ${ }^{\star} \quad$ David Svoboda Martin Maška $^{\star}$ \\ ${ }^{\star}$ Centre for Biomedical Image Analysis, Masaryk University, Brno, Czech Republic \\ ${ }^{\dagger}$ Laboratory of Mathematical Methods of Image Processing, Faculty of Computational \\ Mathematics and Cybernetics, Lomonosov Moscow State University, Moscow, Russia \\ ‡ Institute of Computer Science, Masaryk University, Brno, Czech Republic \\ * Inria, France
}

\begin{abstract}
The existence of benchmark datasets is essential to objectively evaluate various image analysis methods. Nevertheless, manual annotations of fluorescence microscopy image data are very laborious and not often practicable, especially in the case of 3D+t experiments. In this work, we propose a simulation system capable of generating 3D time-lapse sequences of single motile cells with filopodial protrusions, accompanied by inherently generated ground truth. The system consists of three globally synchronized modules, each responsible for a separate task: the evolution of filopodia on a molecular level, linear elastic deformation of the entire cell with filopodia, and generation of realistic, time-coherent cell texture. The capability of our system is demonstrated by generating a synthetic 3D time-lapse sequence of a single lung cancer cell with two growing filopodia, visually resembling its real counterpart acquired using a confocal fluorescence microscope.
\end{abstract}

Index Terms - Simulation, 3D time-lapse sequence, synthetic cell, cell deformation, filopodium evolution

\section{INTRODUCTION}

Filopodia are dynamic bundles of parallel actin filaments protruding forward from the periphery of motile cells [1]. Their typical function is to probe the surrounding environment for cues and promote the locomotion of crawling cells, thus contributing to important physiological processes, such as chemotaxis, angiogenesis, or embryonic development [2].

Modern time-lapse confocal microscopy and fluorescent labeling is a particularly powerful approach to study such processes in vivo [3], calling for robust, quantitative, and ideally fully automatic image analysis [4, 5]. However, an objective assessment of image analysis methods remains an open question. A thorough evaluation requires the availability of reference annotations. Being often prepared manually by human

This work was supported by the Czech Science Foundation under the grant number GA16-03909Y. experts, this leads to a subjective, highly error-prone, and extremely laborious task, especially when it comes to manually annotating 3D time-lapse image data [6].

To address the question of objective evaluation, two recent benchmarks of particle and cell tracking methods $[7,8]$ exploited computer-simulated image datasets, accompanied by inherently generated ground truth. The simulation platforms used in these benchmarks are capable of generating realistic time-lapse sequences of intra-cellular and inter-cellular particles [7] and of motile, nuclearly stained cells [9]. To the best of our knowledge, there are no tools currently available capable of generating 3D time-lapse sequences of motile, cytoplasmically stained cells with filopodial protrusions, although they might help to stimulate the development of filopodium segmentation and tracking routines fully in 3D [10,11].

In this paper, we propose a first simulation system for generating 3D time-lapse sequences of single motile cells with filopodial protrusions. The simulation of filopodium growth is based on a stochastic model implemented using the Gillespie algorithm. The deformation of the entire cell with filopodia is performed using an elastic model. The realistic cell texture is generated by rendering simulated fluorescent particles into the images, submitted afterwards to a virtual microscope. The output generated by the simulation system has the form of a 3D synthetic time-lapse sequence, accompanied by labeled masks of the cell body and individual filopodia. Such a composite output allows one to benchmark filopodium segmentation and tracking algorithms and to study how segmentation and tracking errors can affect quantitative parameters of motile cells with filopodial protrusions, such as the number, length, and lifetime of filopodia or their protrusion and retraction rates.

\section{PROPOSED SIMULATION SYSTEM}

In this section, we describe our simulation system, including the system overview, filopodia growth simulation, filopodia and cell deformation, and realistic texture generation. 


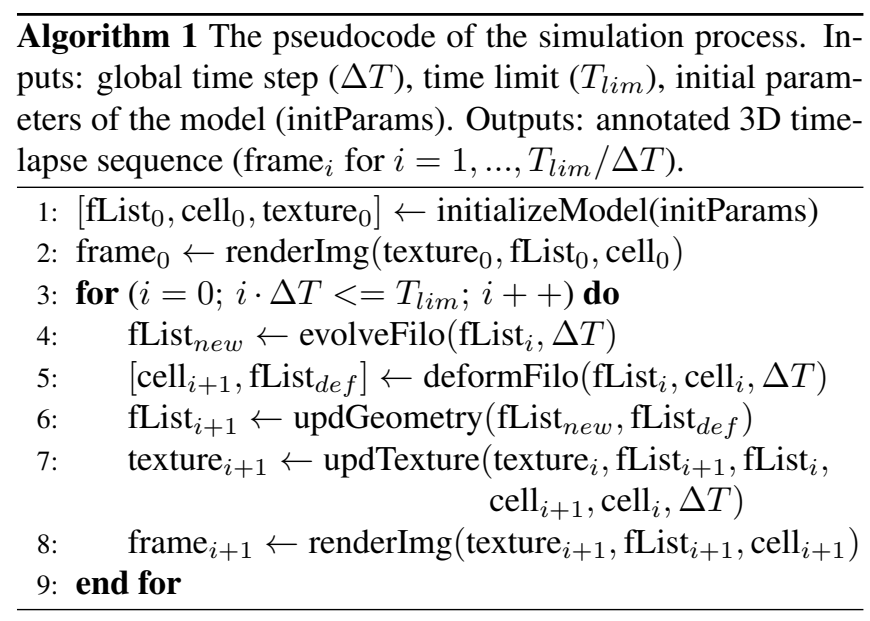

\subsection{System overview}

The simulation of a 3D time-lapse sequence of a single motile cell with filopodial protrusions consists of three independent parts: modeling of filopodium growth, simultaneous deformation of the cell body and individual filopodia, and generation of the realistic cell texture. Each of these three tasks is done by a separate system module: geometry module (GM), deformation module (DM), and texture module (TM). The modules are independent software components and they are executed from the main component, a supervisor, which is responsible for the proper exchange of the data between the modules, setting the parameters, and synchronizing the time.

The whole simulation process (see Alg. 1) starts by initializing the meshes of the cell body (cell ${ }_{0}$ ) and individual filopodia ( fList $_{0}$ ), setting their growth parameters and the positions where they are attached to the cell body, generating the initial cell texture (texture ${ }_{0}$ ), and finally rendering the first frame of the time-lapse sequence $\left(\right.$ frame $\left._{0}\right)$. In our data representation, cell $_{i}$ includes only its geometry stored as a tetrahedral mesh. Each filopodium in fList ${ }_{i}$ is represented by its geometry and growth-related data. The filopodium geometry is stored as a 3D polyline, equipped with a scalar data defining the radius in each node. The growth-related data consists of the parameters and molecule concentrations described in Section 2.2. After the initialization, the simulation process continues iteratively. In each iteration, the morphology of each individual filopodium (i.e., its length and width) is evolved (fList ${ }_{\text {new }}$ ) first. Next, the cell body $\left(\right.$ cell $\left._{i}\right)$ and the filopodia $\left(\mathrm{fList}_{i}\right)$ from the previous iteration are deformed using the pressures applied to the cell body and forces applied to the filopodia. As a result, we obtain the deformed cell body $\left(\operatorname{cell}_{i+1}\right)$ and the deformed filopodia ( fList $_{d e f}$ ) that are subsequently integrated with their actual morphology ( List $_{\text {new }}$ ), resulting in the fully updated filopodia ( fList $_{i+1}$ ). In case a filopodium has grown, the corresponding segment is added to the tip of its mesh. The position of the new segment is obtained by extrapolating the positions of the filopodium nodes in fList $_{d e f}$ in the

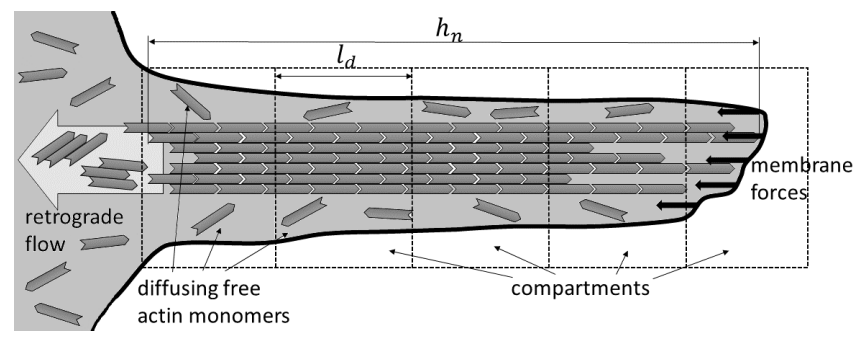

Fig. 1. Filopodium molecular growth model $\left(l_{d}\right.$ is the compartment size, $h_{n}$ is the filament length).

nodes corresponding to the new segment, thus resulting in a smooth continuation of the deformed filopodium. In case the filopodium has shortened, we remove the corresponding segments from its mesh. After the geometry of the whole cell is updated, we update the texture accordingly, which boils down to updating the positions of the simulated fluorescent particles. Finally, the current frame of the sequence $\left(\right.$ frame $\left._{i+1}\right)$ is created by rendering the model.

Since the modules are independent, they can work with their own internal time steps, regularly exporting the particular outputs. A user-defined global time interval $\Delta T$, during which each module is operating, corresponds to the time step between the frames of the resulting time-lapse sequence. The simulation process is terminated after the total accumulated time reaches a pre-defined limit $T_{\text {lim }}$.

The labeled masks of the cell body and individual filopodia, serving as the ground truth for a generated 3D time-lapse sequence, are formed by projecting and rasterizing the meshes of the cell body and individual filopodia to the image domain.

\subsection{Modeling of filopodium growth}

A filopodium is a bundle of $N$ actin filaments enclosed by the cell membrane [1]. Its length is defined as the length of the longest actin filament $h=\max \left(h_{n}\right)$ where $n$ is the actin filament index. Each actin filament consists of actin monomers connected with a half-monomer-size overlap $\delta=2.7 \mathrm{~nm}$ [12] (see Fig. 1). The evolution of each actin filament is affected by four processes: 1) diffusion of free actin monomers along the filopodium; 2) polymerization and depolymerization of actin filaments; 3) fluctuation of the membrane under load, slowing down the polymerization rate of actin filaments; and 4) a constant velocity retrograde flow that continuously pulls actin filaments into the cell body. We modeled these processes on a molecular level using the Gillespie algorithm [12].

The filopodium volume is discretized by dividing it into $L$ compartments, with a compartment length of $l_{d}=50 \mathrm{~nm}$. Let $a_{l}, l=1, \ldots, L$, denote the number of actin monomers in the $l$-th compartment, being indexed from the filopodium tip. The diffusion of each free actin monomer is defined as 1D random walk [13] with reflective boundary conditions at the filopodium tip $\left(P_{1 \rightarrow 0}=0\right)$. At the filopodium base, the 
number of actin monomers is kept unchanged at the constant bulk value $\left(a_{L+1}=5\right)$ as the number of free actin monomers in the cell body is significantly higher than in the filopodium. Since free actin monomers move between neighboring compartments and stay in the same compartment with equal probability, the reaction time of free actin monomer diffusion is given as $\Delta t=\frac{l_{d}^{2}}{3 D}$, where $D$ is the diffusion constant [13].

The actin filament polymerization rate $k_{n}$ depends on the thermal energy of the system and the membrane load force, defined for each individual filament depending on membrane fluctuations [12]. The actin filament depolymerization rate $k_{d}$ does not depend on the membrane load force and has the same value for all filaments.

Taking into account the above mentioned processes, the motion equation for each filament length $h_{n}$ is defined as:

$$
\Delta h_{n}=-\nu_{r e t r} \Delta t+\xi\left(a_{n}, k_{n}, \Delta t\right) \delta-\eta\left(a_{n}, k_{d}, \Delta t\right) \delta
$$

where $\Delta h_{n}$ is the change of filament length over the reaction time $\Delta t, \nu_{\text {retr }}$ is the retrograde flow velocity, and $\xi$ and $\eta$ are the binary indicators of polymerization and depolymerization events, respectively.

Every iteration of the Gillespie simulation involves three steps [14]. First, two random numbers, $r_{1}$ and $r_{2}$, uniformly distributed in the $[0,1]$ interval are generated. Next, the type of event to occur in a particular iteration is established as depolymerization $(\xi=0 ; \eta=1)$ if $0 \leq r_{1}<\frac{k_{d}}{k_{d}+k_{n}}$, polymerization $(\xi=1 ; \eta=0)$ if $\frac{k_{d}}{k_{d}+k_{n}} \leq r_{1}<\frac{k_{n}}{k_{d}+k_{n}}$, or free actin monomer diffusion $(\xi=\eta=0)$ if $r_{1} \geq \frac{k_{n}}{k_{d}+k_{n}}$. Finally, depending on the event type, the number of free actin monomers in each compartment is updated, and the reaction time $\Delta t$ is calculated as either $\Delta t=l_{d}^{2} /(3 D)$ in case of diffusion or $\Delta t=\left(a_{n}^{t i p}\left(k_{d}+k_{n}\right)\right)^{-1} \ln \left(1 / r_{2}\right)$ in case of polymerization or depolymerization, with $a_{n}^{t i p}$ being the number of free actin monomers in the filopodium compartment that corresponds to the tip of the $n$-th filament, not necessarily being the first one.

The Gillespie simulations are performed individually for each actin filament over the global time step $\Delta T$. The resulting geometry changes are transmitted to the deformation and texture modules of the simulation system.

The 3D filopodium shape is modeled as a Gaussian with $\sigma$ corresponding to the number of filaments. The radii at the points between compartments (nodes) are set accordingly.

\subsection{Deformation of cell body and filopodia}

To mimic realistic deformations, the cell body and the filopodia are modeled as elastic objects coupled together via mechanical constraints. For the both types of objects, the corotational finite element formulation of the linear elasticity is employed. This approach is computationally efficient and at the same time, it allows for correct simulation of large deformations [15]. Therefore, the mechanical response of both cell body and filopodia to given applied forces and pressures is determined by Young's modulus and Poisson's ratio, which can be set independently for each object instance. While the same mathematical formulation is used for all simulated objects, different types of elements are employed for their discretization. The cell body is discretized by a volume mesh composed of linear tetrahedra: each element is given by four nodes having three positional degrees of freedom. As in [16], random pressures can be applied to the surface of this mesh, imposing motion and deformation of the cell body. Since the geometry of filopodia is given by line segments equipped with radii, we opted for linear beam elements based on the Timoshenko formulation [17]. While being computationally efficient, the beam elements allow for modeling of elongation and bending, which are two basic types of deformations needed for realistic simulation of filopodial movement. Unlike tetrahedral elements, the beam is given by two nodes, each having six degrees of freedom: positions and orientations necessary to model the bending. It is therefore possible to apply linear and rotational forces (torques) to the nodes of filopodia.

Each filopodium is mechanically attached to the cell body: it is supposed that initially the base of each filopodium is located inside a tetrahedron that belongs to the cell body mesh. The initial position and orientation of the base with respect to the corresponding tetrahedron is then kept constant during the entire simulation step $\Delta T$ similarly as in [18]. Therefore, the motion of the cell body due to the applied surface pressures results in the motion of the filopodium via the motion of its base. Vice versa, a motion of the filopodium is propagated to the cell body via the tetrahedron associated to the filopodium base. Numerically, the coupling between the objects is implemented using bilateral constraints based on Lagrange multipliers: in every internal step of the deformation module, first the dynamics of each object (i.e., every filopodium and the cell body) is computed independently. Then, this predictive motion is corrected by applying the constraints that maintain the initial positions and orientations of filopodium bases with respect to the cell body tetrahedra. The implicit Euler solver is used for time integration, which requires solution of the local system of linear equations corresponding to each object. For the cell body, the solution is computed by a solver based on the LDL-decomposition, whereas a solver based on the Thomas algorithm for block tridiagonal matrices is employed for each filopodium [19].

\subsection{Generation of cell texture}

The cell texture is represented as a set of many simulated fluorescent particles placed into a phantom image of a simulated cell. In particular, a floating-point precision position is stored with every particle to represent its real world coordinate, regardless of a desired resolution of created images. When rendering successive frames of the generated sequence, the particle positions follow the cell geometry changes by tracking and interpolating displacements of the mesh vertices. The intensity assigned to a phantom image voxel is proportional to the 

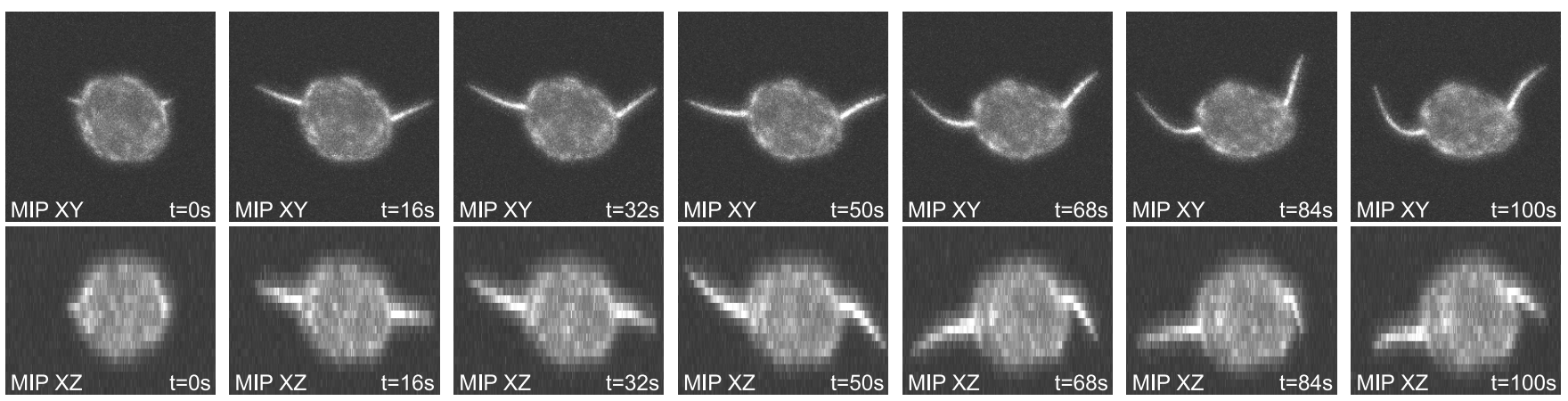

Fig. 2. Two maximum intensity projections of the resulting 3D time-lapse sequence. The voxel size is $0.126 \times 0.126 \times 1.0 \mu \mathrm{m}$.
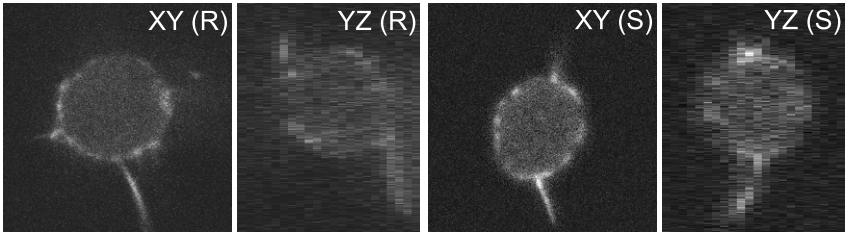

Fig. 3. An example of XY and YZ slices of real (R) and synthetic $(\mathrm{S})$ images. The voxel size is $0.126 \times 0.126 \times 1.0 \mu \mathrm{m}$.

number of particles that are projected into the voxel volume. The initial distribution of particles is sampled from an image with an isotropic mosaic of Perlin noises, and is restricted to the cell volume. Every voxel introduces multiple particles at the same respective coordinate, the number of them is proportional to the voxel intensity. For each filopodium, the particles are placed on its skeleton in amounts proportional to the local radius of the filopodium.

To obtain the final image that is visually similar to a real microscopy one, the phantom image is submitted to a virtual microscope [20]. In particular, the phantom image is blurred by an experimental point spread function of a real optical system, affected by an uneven illumination phenomenon, resampled to the resolution as that of the real microscope setup, and degraded by shot noise, dark current, and read-out noise.

\section{RESULTS}

To demonstrate the capability of our simulation system, we focused on a motile lung cancer cell with two filopodial protrusions. As a reference, we considered real image data with the voxel size of $0.126 \times 0.126 \times 1.0 \mu \mathrm{m}$, being acquired using a spinning disk confocal microscope equipped with a water Plan-Apo $63 \times / 1.20$ objective lens [21]. The initial geometry of the simulated cell body was taken from the real image data. The bases of both filopodia were randomly placed on the cell body surface. For both filopodia, their growth parameters were taken from [12] and their growth dynamics were regulated by random retrograde flow velocities of 45 to $55 \mathrm{~nm} / \mathrm{s}$.
The cell body was deformed by random pressures generated similar to [16]. The filopodia were deformed by applying random forces of 0.5 to $1 \mathrm{mN}$ to their tips. The Young's modulus for the cell body and filopodia was set to $1 \mathrm{kPa}$ and $50 \mathrm{kPa}$, respectively, reflecting the fact that filopodia are stiffer than the cell body [1]. The Poisson's ratio for both the cell body and filopodia was fixed at 0.4. The global time step and time limit were fixed at $\Delta T=2 \mathrm{~s}$ and $T_{\text {lim }}=100 \mathrm{~s}$, respectively, yielding a time-lapse sequence of 51 frames long. The synthetic intensity profiles were adapted to visually imitate the real ones by scaling and shifting constants. Furthermore, factoring the cell body volume by utilizing a mosaic of Perlin noises during the cell texture initialization allowed us to introduce the more pronounced cell structures found near the outer cell membrane, and the membrane itself. Approximately nine million particles for the cell body and a quarter of million for both filopodia contributed to each phantom image.

The resulting 3D time-lapse sequence generated with the described parameters is shown in Fig. 2, demonstrating the growth and motion of filopodia. To compare visually the synthetic cell texture with its real counterpart, an example of XY and YZ slices of both is shown in Fig. 3.

\section{CONCLUSION}

We have presented a novel approach to generating synthetic 3D time-lapse sequences of single motile cells with filopodial protrusions. The proposed approach utilizes a stochastic model for the simulation of filopodium growth on a molecular level. The motion and deformation of the cell is modeled using the linear elasticity. The cell texture is composed of a large number of simulated fluorescent particles, initially sampled from a mosaic of Perlin noises and updated according to the cell geometry changes. As a proof of the concept, we generated a 3D time-lapse sequence of a single lung cancer cell with two growing filopodia, showing coherent physic-laws constrained shape changes followed by coherently developing texture. In future work, we intend to adapt the simulation parameters across different cell phenotypes, including those with filopodium branching and interaction with one another. 


\section{REFERENCES}

[1] M.-F. Carlier, Actin-based Motility, Springer-Verlag, Heidelberg, Germany, 2010.

[2] P. K. Mattila and P. Lappalainen, "Filopodia: Molecular architecture and cellular functions," Nat. Rev. Mol. Cell Biol., vol. 9, no. 6, pp. 446-454, 2008.

[3] C. Ortiz-de-Solórzano, A. Muñoz-Barrutia, E. Meijering, and M. Kozubek, "Toward a morphodynamic model of the cell," Signal Processing Magazine, vol. 32, no. 1, pp. 20-29, 2015.

[4] A. Cardona and P. Tomancak, "Current challenges in open-source bioimage informatics," Nature Methods, vol. 9, no. 7, pp. 661-665, 2012.

[5] A. E. Carpenter, L. Kamentsky, and K. W. Eliceiri, "A call for bioimaging software usability," Nature Methods, vol. 9, no. 7, pp. 666-670, 2012.

[6] D. L. Coutu and T Schroeder, "Probing cellular processes by long-term live imaging-historic problems and current solutions," Journal of Cell Science, vol. 126, no. 17, pp. 3805-3815, 2013.

[7] N. Chenouard, I. Smal, F. de Chaumont, M. Maška, et al., "Objective comparison of particle tracking methods," Nature Methods, vol. 11, no. 3, pp. 281-289, 2014.

[8] M. Maška, V. Ulman, D. Svoboda, et al., "A benchmark for comparison of cell tracking algorithms," Bioinformatics, vol. 30, no. 11, pp. 1609-1617, 2014.

[9] D. Svoboda and V. Ulman, "Mitogen: A framework for generating 3D synthetic time-lapse sequences of cell populations in fluorescence microscopy," IEEE Trans. Med. Imag., vol. 36, no. 1, pp. 310-321, 2017.

[10] D. Tsygankov, C. G. Bilancia, E. A. Vitriol, K. M. Hahn, M. Peifer, and T. C. Elston, "CellGeo: A computational platform for the analysis of shape changes in cells with complex geometries," Journal of Cell Biology, vol. 204, no. 3, pp. 443-460, 2014.

[11] D. J. Barry, C. H. Durkin, J. V. Abella, and M. Way, "Open source software for quantification of cell migration, protrusions, and fluorescence intensities," Journal of Cell Biology, vol. 209, no. 1, pp. 163-180, 2015.

[12] Y. Lan and G. A. Papoian, "The stochastic dynamics of filopodial growth," Biophysical Journal, vol. 94, no. 10, pp. 3839-3852, 2008.

[13] R. Erban and J. S. Chapman, "Reactive boundary conditions for stochastic simulations of reaction-diffusion processes," Physical Biology, vol. 4, no. 1, pp. 16, 2007.
[14] R. Erban, J. S. Chapman, and P. Maini, "A practical guide to stochastic simulations of reaction-diffusion processes," arXiv preprint arXiv:0704.1908, 2007.

[15] C. A. Felippa, "A systematic approach to the element independent corotational dynamics of finite elements," Tech. Rep. CU-CAS-00-03, Cent. for Aerosp. Str., 2000.

[16] D. Svoboda, V. Ulman, and I. Peterlík, "On proper simulation of chromatin structure in static images as well as in time-lapse sequences in fluorescence microscopy," in IEEE Int. Symp. Biomed. Imag., 2015, pp. 712-716.

[17] J. S. Przemieniecki, "Matrix structural analysis of substructures," AIAA J., vol. 1, no. 1, pp. 138-147, 1963.

[18] I. Peterlík, C. Duriez, and S. Cotin, "Modeling and realtime simulation of a vascularized liver tissue," in Int. Conf. MICCAI, 2012, pp. 50-57.

[19] S. Cotin, C. Duriez, J. Lenoir, P. Neumann, and S. Dawson, "New approaches to catheter navigation for interventional radiology simulation," in Int. Conf. MICCAI, 2005, pp. 534-542.

[20] D. Svoboda, M. Kozubek, and S. Stejskal, "Generation of digital phantoms of cell nuclei and simulation of image formation in 3D image cytometry," Cytometry Part A, vol. 75A, no. 6, pp. 494-509, 2009.

[21] M. Maška, X. Morales, A. Muñoz-Barrutia, A. Rouzaut, and C. Ortiz-de-Solórzano, "Automatic quantification of filopodia-based cell migration," in IEEE Int. Symp. Biomed. Imag., 2013, pp. 668-671. 